\title{
Study Terminated
}

National Cancer Institute

\section{Source}

National Cancer Institute. Study Terminated. NCI Thesaurus. Code C70757.

A clinical study status designating the study has been stopped prematurely and will not resume. Subject enrollment stopped. No participants are treated or being followed up. 